\title{
Lumbar Disc Screening Using Back Pain Questionnaires: Oswestry Low Back Pain Score, Aberdeen Low Back Pain Scale, and Acute Low Back Pain Screening Questionnaire
}

\author{
Do Yeon Kim¹, Chang Hyun $\mathrm{Oh}^{2}$, Seung Hwan Yoon ${ }^{1}$, \\ Hyung Chun Park ${ }^{1}$, Chong Oon Park ${ }^{1}$ \\ ${ }^{I}$ Department of Neurosurgery, Inha University College of Medicine, Incheon, \\ ${ }^{2}$ Department of Neurosurgery, Seoul Regional Military Manpower Administration, Seoul, Korea
}

\begin{abstract}
Objective: To evaluate the usefulness of back pain questionnaires for lumbar disc screening among Korean young males. Methods: We carried out a survey for lumbar disc screening through back pain questionnaires among the volunteers with or without back pain. Three types of back pain questionnaire (Oswestry Low Back Pain Score, Aberdeen Low Back Pain Scale, and Acute Low Back Pain Screeing Questionnaire) were randomly assigned to the examinees. The authors reviewed lumbar imaging studies (simple lumbar radiographs, lumbar computed tomography, and magnetic resolutional images), and the severity of lumbar disc herniation was categorized according to the guidelines issued by the Korean military directorate. We calculated the relationship between the back pain questionnaire scores and the severity of lumbar disc herniation. Results: The scores of back pain questionnaires increased according to the severity of lumbar disc herniation. But, the range of scores was very vague, so it is less predictable to detect lumbar disc herniation using only back pain questionnaires. The sensitivity between the back pain questionnaires and the presence of lumbar disc herniation was low (16-64\%).

Conclusion: Screening of lumbar disc herniation using only back pain questionnaires has limited value.
\end{abstract}

Key Words: Mass Screening $\cdot$ Intervertebral Disc Displacement $\cdot$ Korea $\cdot$ Low Back Pain $\cdot$ Questionnaires

\section{INTRODUCTION}

There are many types of back pain questionnaires. There are at least 24 methods including regional or private ${ }^{1)}$. However, most of the back pain questionnaires are used for post treatment follow up rather than for diagnostic purpose. Besides this, research regarding the relation between back pain questionnaires on the references and actual spinal disease are insufficient. Therefore the authors translated three types of back pain questionnaires into Korean and then undertook a survey targeting examinees of the physical examination for the military draft, to allow an evaluation of the relationship between the results of the three back pain questionnaires and actual spinal disease, particularly lumbar disc herniation, as

- Received: April 30, 2012 - Revised: August 14, 2012

- Accepted: September 25, 2012

Corresponding Author: Seung Hwan Yoon, MD

Department of Neurosurgery, Inha University Hospital, 7-206, 3-Ga,

Sinheung-dong, Jung-gu, Incheon 400-711, Korea

Tel: +82-32-890-2370, Fax: +82-32-890-2947

Email: nsyoon@gmail.com well as its usefulness as a screening test for disc herniation.

\section{MATERIALS AND METHODS}

The Republic of Korea is one of the nations that have compulsory conscription. All 18 year old Korean males must undertake military duty if they do not have a serious mental or physical disability, so they have to do a physical examination for the military draft. The participants in this research are 223 young males who voluntarily participated after they had been informed of the purpose of this research among examinees of the physical examination for the military draft in Seoul Regional Military Manpower Administration between March 2010 and July 2011.

The participants got a basic questionnaire and three types of back pain questionnaire. The basic questionnaire includes an explanation that this survey does not have any influence on the military test result, whether they exercised when they were high school students, family history of back pain, whether they had had any accidents, thoughts about their own health, whether they smoke, whether they have back pain or radiating 
pain and how they think about their own waist health. The three types of back pain questionnaire were Oswestry Low Back Pain Score (Oswestry), Aberdeen Low Back Pain Scale (Aberdeen) and Acute Low Back Pain Screeing Questionnaire (ALBPSQ) translated into Korean, and they were randomly applied to the participants. The categories related to sexual activity were deleted due to the participants being 19 year old boys, and the some expressions were changed into terms that Koreans are familiar with, for example, mile into meter. The severity of lumbar disc herniation was categorized according to the guidelines issued by the Korean military directorate 702 and 728 (Table 1). Both directorates categorize Status of lumbar disc herniation by Normal disc, Mild disc bulging without com- pression of thecal sac, Disc protrusion or extrusion without compression of thecal sac, Disc protrusion or extrusion with compression of thecal sac, Disc protrusion or extrusion with spinal canal compromise more than 50\%, and Disc protrusion or extrusion with positive cut-off sign. In this research, the severities of lumbar disc herniation were categorized by mild and sever as written in Table 1. lumbar plain radiograph was operated for basic test of imaging study, and Lumbar spine CT and lumbar spine MRI were confirmed by all of the examinees. All imaging studies were read primilily by one radiologist, and then were reread by another neurosurgeon. When there were disagreed in reading, then the radiologist and the neurosurgeon read after consultation. Yet if they do not come

Table 1. The categories of spinal disc herniation according to the Korean military service law

\begin{tabular}{|c|c|c|}
\hline Status of lumbar disc herniation & Physical grade & Severity \\
\hline Normal disc & 1 & \multirow{2}{*}{ Normal } \\
\hline Mild disc bulging without compression of thecal sac & 2 & \\
\hline Disc protrusion or extrusion without compression of thecal sac & 3 & \multirow{2}{*}{ Mild } \\
\hline Disc protrusion or extrusion with compression of thecal sac & 4 & \\
\hline Disc protrusion or extrusion with spinal canal compromise more than $50 \%^{+}$ & 5 & \multirow{2}{*}{ Severe } \\
\hline Disc protrusion or extrusion with positive cut-off sign $^{\ddagger}$ & 5 & \\
\hline
\end{tabular}

"Positive result of electromyogram test was defined as matched radiculopathy findings according to a suspicious level of herniation; ${ }^{+}$spinal canal stenosis was defined as the narrowing of the spinal canal to more than $50 \%$ of mid-sagittal diameter (front to back) in the same plane of imaging study; ${ }^{\dagger}$ cut-off sign was defined as total loss of neuromuscular signals at an interesting level using imaging study.

Table 2. Demographic data of subjects enrolled in this study

\begin{tabular}{|c|c|c|c|c|c|}
\hline Category & Sub & Total (\%) & Normal Disc Group & $\begin{array}{l}\text { Herniated Lumbar } \\
\text { Disc Group }\end{array}$ & p-value \\
\hline No. & & 223 & 66 & 157 & \\
\hline Age (yr) & & 20, [18-32] & 20, [18-28] & 20, [18-32] & \\
\hline Sex & & Male & Male & Male & \\
\hline Physical Exercise & Yes & $100(44.8)$ & 23 & 77 & 0.052 \\
\hline Physical Exercise & No & $123(55.2)$ & 43 & 80 & \\
\hline Back Pain of Family Member & Yes & $97(43.5)$ & 23 & 74 & 0.092 \\
\hline Back Pain of Family Member & No & $126(56.5)$ & 43 & 83 & \\
\hline History of Lumbar Accident & Yes & $105(47.1)$ & 23 & 82 & 0.014 \\
\hline History of Lumbar Accident & No & $118(52.9)$ & 43 & 75 & \\
\hline Mind about one's Health & I'm healthy & $102(45.7)$ & 35 & 67 & 0.158 \\
\hline Mind of Own's Health & I'm not healthy & $121(54.3)$ & 31 & 90 & \\
\hline Smoking & Yes & $101(45.3)$ & 34 & 67 & 0.228 \\
\hline Smoking & No & $122(54.7)$ & 32 & 90 & \\
\hline Back Pain & Yes & $211(94.6)$ & 57 & 154 & $<0.001$ \\
\hline Back Pain & No & $12(5.4)$ & 9 & 3 & \\
\hline Radiating Pain & Yes & $161(72.2)$ & 38 & 123 & 0.001 \\
\hline Radiating Pain & No & $62(27.8)$ & 28 & 34 & \\
\hline Diagnosis of Lumbar Disc & Diagnosed by physician & $123(55.2)$ & 13 & 110 & $<0.001$ \\
\hline Diagnosis of Lumbar Disc & Suspected by himself & 43 (19.3) & 24 & 19 & \\
\hline Diagnosis of Lumbar Disc & Not suspected by himself & $57(25.5)$ & 29 & 28 & \\
\hline Back Pain Questionnaires & Oswestry & $76(34.1)$ & 24 & 52 & 0.733 \\
\hline Back Pain Questionnaires & Aberdeen & $73(32.7)$ & 23 & 50 & 0.506 \\
\hline Back Pain Questionnaires & ALBPSQ & 74 (33.2) & 19 & 55 & 0.415 \\
\hline
\end{tabular}


to an agreement, then electromyogram (EMG) was operated for checking thecal sac compression due to disc herniation.

For statistical analysis, SPSS 12.0 Korean version was used, and for analysis the relations between the basic questionnaires and back pain related questionnaires, statistical techniques such as the Student T-test and others were applied. The p-value level was set at under 0.05 for significance test.

\section{RESULTS}

The basic data of all 223 examinees were written in Table 2. The average age was 20 , the spread was 18 to 29 , and all examinees were male (67.7\% were 18 years old).

Normal discs were found in 66, mild and severe disc herniation were found in 157, a family history of back pain was found in 43.5\%, and Statistical significance was not found in the relationship between the normal disc group and disc herniation ( $p$-value 0.052, 0.092). An accident which impacted on the back was found in $47.1 \%$, and was found frequently in the disc herniation group $(\mathrm{p}=0.014)$. There was no statistical significance between the ideas about their own health and their smoking habits (p-value 0.158, 0.228). Statistically significant differences were shown between the normal disc group and disc herniation group regarding having back pain, radiating pain and the thoughts about their own back health $(\mathrm{p}<0.001)$. Oswestry, Aberdeen, and ALBPSQ questionnaires were applied to 76, 73 and 74 males respectively, and there were no statistical differences between the groups.

The standard points of Oswestry Low Back Pain were 0-45 (excluding sexual category), the points were spread from 0 to 42 (Fig. 1). In the normal disc group, the distribution of the points of Oswestry were $9.25 \pm 9.34$, and this showed statistically significant differences from the distribution of the disc herniation group, $15.00 \pm 9.48(\mathrm{p}=0.016)$. There were no statistically significant differences between the normal disc group and mild disc herniation group $(p=0.878)$, but the severe disc herniation group showed increasing point of view compared with all of the normal and mild disc herniation groups ( $\mathrm{p}=$ $0.004,0.018)$. The Oswestry points were spread widely between the normal disc and Disc herniation group (Fig. 1B), and it was difficult to judge if there was disc herniation or not based on the mid-point of Oswestry (Table 3, peculiarity 16.4\%, sensitiveness 95.2\%).

The standard for the Aberdeen Low Back Pain Scale was 0-72 (excluding the sexual category), the points were spread from 0 to 49 (Fig. 2). In the normal disc group, the distribution was $12.39 \pm 10.24$, and this showed statistically significant differences with the distribution of the disc herniation group, 24.12 \pm 13.36 ( $p<0.001)$. There were no statistically significant differences between the normal disc group and mild disc herniation group $(p=0.076)$, although the severe disc herniation group showed increasing points compared with all of the normal and mild disc herniation groups $(\mathrm{p}<0.001,0.034)$. Yet,
Oswestry

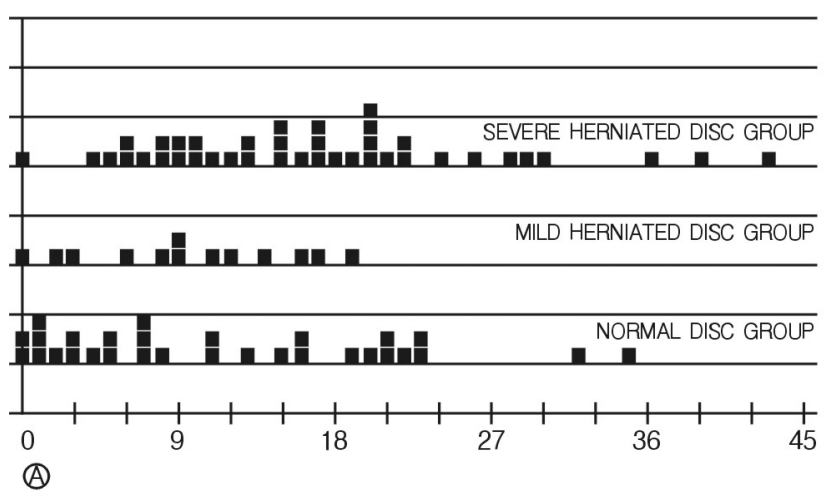

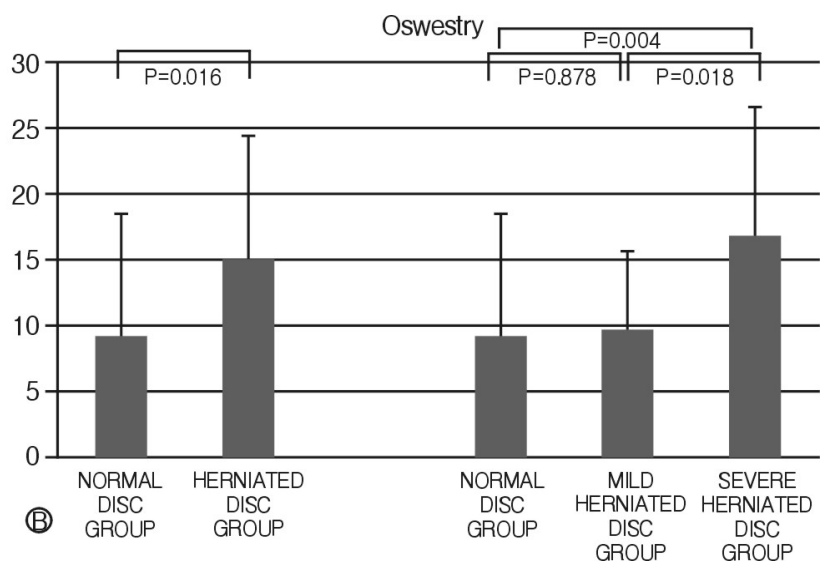

Fig. 1. Oswestry low back pain score according to the presence or the severity of lumbar disc herniation.

Table 3. Oswestry low back pain score according to the severity of lumbar disc herniation

\begin{tabular}{|c|c|c|c|c|c|}
\hline Oswestry low back & pain scores & Normal disc group (\%) & Mod. disc herniation (\%) & Severe disc herniation (\%) & Total \\
\hline Bisection of scores & $0-22$ & $20(30.3)$ & $14(21.2)$ & $32(48.5)$ & 66 \\
\hline Bisection of scores & $23-45$ & $1(10.0)$ & $1(10.0)$ & $8(80.0)$ & 10 \\
\hline Trisection of scores & $0-15$ & $17(35.4)$ & $11(22.9)$ & $20(41.7)$ & 48 \\
\hline Trisection of scores & $16-30$ & $3(13.0)$ & $3(13.0)$ & $17(74.0)$ & 23 \\
\hline Trisection of scores & $31-45$ & $1(20.0)$ & $1(20.0)$ & $3(80.0)$ & 5 \\
\hline
\end{tabular}


as well as Oswestry, the Aberdeen scale were also spread widely according to disc herniation grade, so it was not easy to judge if there was disc herniation or not based on the points only (Fig. 2B). The sensitiveness was 50.0\%, and peculiarity was $95.2 \%$ in a test which divided by the intermediate value of the minimum value of Aberdeen scale and the maximum scale (Table 4).

The standard of the Acute Low Back Pain Screening Questionnaire was 12-203 (excluding the sexual category), the points were spread from 29-169 (Fig. 3). In the normal disc group, the distribution was $83.37 \pm 42.77$, and this showed statisti- cally significant differences with the distribution of the disc herniation group, $109.09 \pm 36.45(\mathrm{p}<0.013)$. There were no statistically significant differences between the normal disc group and mild disc herniation group ( $p=0.321,0.054$ ), although the severe disc herniation group showed increasing points compared with the normal disc group $(p=0.002)$. Yet, it was not easy to judge if there was disc herniation or not based on the ALBPSQ scale only (Fig. 3B). The peculiarity was $73.3 \%$, and sensitiveness was $64.4 \%$ in a test which divided by the intermediate value (Table 5).

The ratio of the severity of lumbar disc herniation was cal-
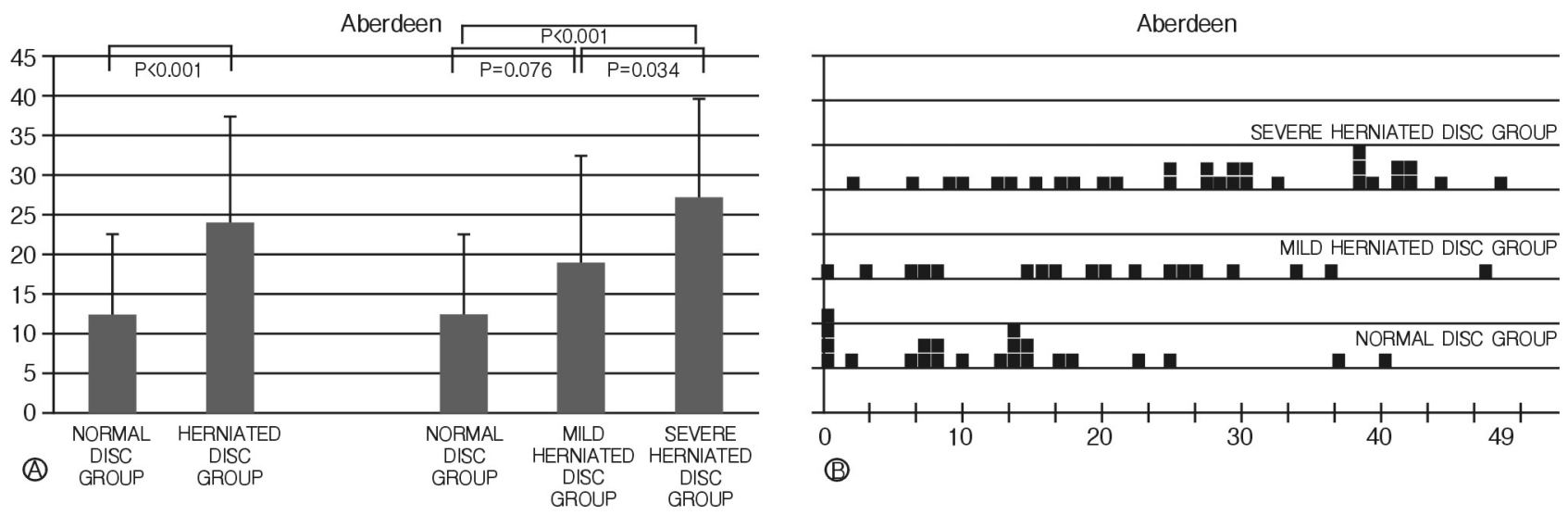

Fig. 2. Aberdeen low back pain scale according to the presence or the severity of lumbar disc herniation.

Table 4. Aberdeen low back pain scale scores according to the severity of disc herniation

\begin{tabular}{lrcrrr}
\hline \hline Aberdeen Low Back Pain Scale & & Normal disc group (\%) & Mod. disc herniation (\%) & Severe disc herniation (\%) & Total \\
\hline Bisection of scores & $0-24$ & $15(35.0)$ & $14(32.5)$ & $6(20.0)$ & $14(32.5)$ \\
Bisection of scores & $25-49$ & $2(6.7)$ & $11(29.8)$ & $22(73.3)$ & $12(32.4)$ \\
Trisection of scores & $0-18$ & $14(37.8)$ & $7(30.4)$ & $13(56.5)$ & 30 \\
Trisection of scores & $19-36$ & $3(13.0)$ & $2(15.4)$ & $11(84.6)$ & 13 \\
Trisection of scores & $37-49$ & $0(0.0)$ & &
\end{tabular}
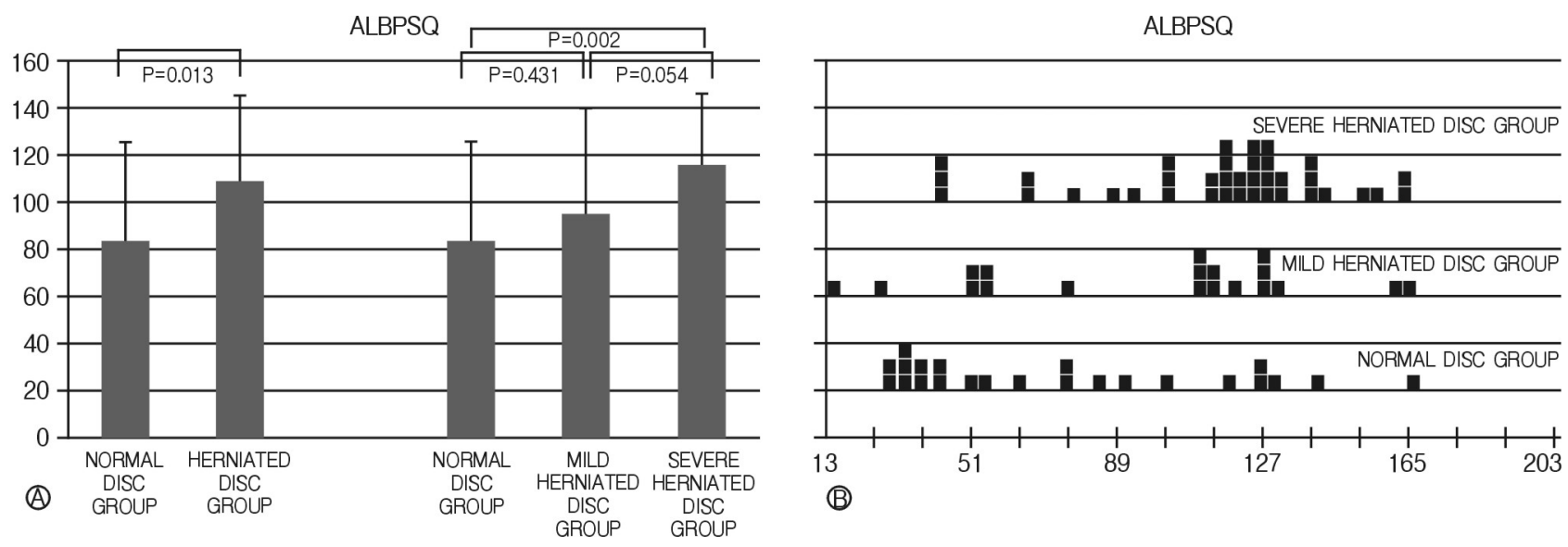

Fig. 3. Acute lower back pain screening questionnaire results according to the presence or the severity of lumbar disc herniation. 
Table 5. Acute lower back pain screening questionnaire scores according to the severity of disc herniation

\begin{tabular}{lccrr}
\hline \hline Acute low back pain screening questionair score & Normal disc group (\%) & Mod. disc herniation (\%) & Severe disc herniation (\%) & Total \\
\hline Bisection of scores & $13-108$ & $11(34.4)$ & $10(31.2)$ & $11(34.4)$ \\
Bisection of scores & $109-203$ & $4(9.5)$ & $11(26.2)$ & 32 \\
Trisection of scores & $13-76$ & $9(40.9)$ & $8(36.4)$ & $27(64.3)$ \\
Trisection of scores & $77-140$ & $5(12.5)$ & $10(25.0)$ & $5(22.7)$ \\
Trisection of scores & $141-203$ & $1(8.3)$ & $3(25.0)$ & 22 \\
\hline
\end{tabular}

culated by bisection or trisection of scores. In all of the back pain questionnaires, as the scale increased, the ratio of normal discs decreased and severe discs increased. However, based on the trisection of the scores, the ratio of severe disc herniation (Oswestry 41.7\%, Aberdeen 32.4\%, ALBPSQ 22.7\%) in the low scale was relatively high.

\section{DISCUSSION}

Back pain is caused by various diseases and the prognosis is affected a lot by socio-psychological factors ${ }^{5)}$. The causes of back pain are various organic lesions in the patients, however disc herniation is the most common cause. Many types of back pain questionnaires are used regionally or privately, and most of the back pain questionnaires are used for post treatment follow up rather than for diagnostic purposes ${ }^{1)}$. In this study, the Oswestry Low Back Pain Score, Aberdeen Low Back Pain Scale and Acute Low Back Pain Screening Questionnaire were applied to participants to check the relationship between the results of the back pain questionnaires and lumbar disc herniation.

The Oswestry Low Back Pain Score is composed of questions in 10 categories about daily activities which can be affected by back pain. In each category, people are made to choose one out of six options; from 0 points (no problem) to 5 points $\left(\right.$ cannot do it) ${ }^{8}$. The maximum point for each question is 5 points, and the result of the division of the summation of all 10 categories by 50 points can be expressed as a percentage. Therefore, Oswestry scores can be categorised from 0 points: no problem, to 100 points: most disabled state. However, in this study, the score was analyzed as it was. The Oswestry test is focused on evaluating physical activity such as standing, walking, lifting, sitting, lying and personal hygiene. This test is generally used for evaluation and improvement by the Medical Re- search Council group, and its usefulness is confirmed by other back pain disordered questionnaires ${ }^{11)}$. It reacts sensitively with small clinical changes ${ }^{9,13)}$, and is verified as good index for analyzing the result of an operation for the spinal fusion patient using the Short Form 36, $2,3,7$.

The Aberdeen lower back pain scale is used for early diag- nosis and post treatment follow up ${ }^{12)}$. It consists of categories of 19 disease-related questions (in this study, 18 excluding sexually related questions) such as taking analgesics, pain causing factors, dispersion of symptoms and the effect of pain on physical activity etc, and each group of questions are subdivided and calculated into several sets, such as left and right, area of pain and parts losing strength etc. The method of calculation is to get different points in each category and to get points double checked. The score is expressed as a percentage as well as the Oswestry, yet in this study, the score was analyzed as it was. Rocchi et al reported that the Aberdeen test has excellent credibility and sensitiveness ${ }^{10)}$.

The Acute Low Back Pain Screening Questionnaire is made up of 3 basic questions and 21 score-based pain related questions. Unlike other questionnaires, this self back pain score questionnaire is composed of categories which have a plus and minus score. The surveyee answers questions on the severity of back pain from 1 to 10 in each category (each category can be double checked in 5 categories), and the total scores are distributed between 20 to 210 points. The questions are focused on daily life disorders rather than symptoms related to pain. ALBPSQ is confirmed as showing the relationship between pain and disabilities by Hurley et $\mathrm{al}^{6}$.

The 9 self questionnaires for evaluating disorders due to back pain (Oswestry, Quebec, Bournemouth, Aberdeen, GreenoughFraser, Stratford, Wadell-Main, Roland-Morris, Von Korff) show excellent credibility and sensitiveness in the research of Rocchi et $\mathrm{al}^{10)}$. The sensitiveness of Oswestry, Aberdeen, ALBPSQ were confirmed highly as well in this study (95.2, 88.2, 73.3 $\%)$, but their peculiarity were shown to be low $(16.4,50.0$, $64.4 \%)$. The score of pain showed increasing statistical significance as the severity of each disc increased, however, because the pain scores were distributed widely, it was impossible to confirm disc herniation or not based on the pain scales only (Fig. 1-3). This example is shown largely though literature reviews. Grotle et al researched 36 kinds of back pain questionnaires based on MEDLINE and only a few of them were confirmed as to their usefulness ${ }^{4}$. Moreover Davidson et al mentioned that back pain questionnaires in SF-36 lack credibility and sensitiveness when applied clinically. 


\section{CONCLUSION}

Back pain related questionnaires: Oswestry Low Back Pain Score, Aberdeen Low Back Pain Scale, and Acute Low Back Pain Screening Questionnaires show meaningful increase in the disc herniation group compared to the normal disc group. However screening tests for disc herniation though back pain related questionnaires lacks credibility and peculiarity.

\section{REFERENCES}

1. Davidson M, Keating JL: A comparison of five low back disability qeustionaires: reliability and responsiveness. Phys Ther 82: 8-24, 2002

2. Demoulin C, Ostelo R, Knottnerus JA, Smeets RJ: Quebec back pain disability scale was responsive and showed reasonable interpretability after a multidisciplinary treatment. J Clin Epidemiol 63:1249-1255, 2010

3. Glassman S, Gornet MF, Branch C, Polly DJr, Peloza J, Schwender JD, et al: MOS short form 36 and oswestry disability index outcomes in lumbar fusion: a multicenter experience. Spine J 6:21-26, 2006

4. Grotle M, Brox JI, Vøllestad NK: Functional status and disability questionnaires: what do they assess? A systematic review of back-specific outcome questionnaires. Spine (Phila Pa 1976) 30:130-140, 2005

5. Hong CK, Park CK, Park HC, Yoon SH: Prevalence and clinical characteristics of intervertebral disc herniation in adolescence: a study based on examination for conscription. Korean J Spine 1:83-87, 2004

6. Hurley DA, Dusoir TE, McDonough SM, Moore AP, Baxter GD: How effective is the acute low back pain screening questionnaire for predicting 1-year follow-up in patients with low back pain? Clin J Pain 17:256-263, 2001

7. Niskanen RO: The Oswestry low back pain disability question naire. a two-year follow-up of spine surgery patients. Scand J Surg 91:208-211, 2002

8. Ostelo RW, de Vet HC: Clinically important outcomes in low back pain. Best Pract Res ClinRheumatol 19(4):593-607, 2005

9. Ostelo RW, Deyo RA, Stratford P, Waddell G, Croft P, Von Korff $M$, et al: Interpreting change scores for pain and functional status in low back pain: towards international consensus regarding minimal important change. Spine (Phila Pa 1976) 33: 90-94, 2008

10. Rocchi MB, Sisti D, Benedetti P, Valentini M, Bellagamba S, Federici A: Critical comparison of nine different selfadministered questionnaires for the evaluation of disability caused by low back pain. EuraMedicophys 41:275-281, 2005

11. Roland M, Fairbank J. The roland-morrisdisability questionnaire and the Oswestry disability questionnaire. Spine (Phila Pa 1976) 25:3115-3124, 2000

12. Ruta DA, Garratt AM, Wardlaw D, Russell IT: Developing a valid and reliable measure of health outcome for patients with low back pain. Spine 19:1887-1896, 1994

13. van der Roer N, Ostelo RW, Bekkering GE, van Tulder MW, de Vet HC: Minimal clinically important change for pain intensity, functional status, and general health status in patients with nonspecific low back pain. Spine (Phila Pa) 31:578-582, 2006 